\title{
Clinical risk management and child mental health
}

\author{
Fiona Subotsky
}

Abstract Risk management is a requirement of clinical governance and a new paradigm for child and adolescent mental health services. Issues are different from those of adult services and a wide range need to be considered, which include not only the risk of harm to self, to others and from others but also from the system (by omission or commission) and to the staff. Systematic policy development, using information from audits, complaints, incidents and inquiries, will be helpful and interagency agreements necessary to promote coordination. The complex regulatory framework is discussed.

Child and adolescent mental health services (CAMHS) are managed in different ways in the UK, but are subject to the clinical governance arrangements, which include management of clinical risk. In adult mental health services, the primary 'risk' focus has been on violence and self-harm in the context of severe mental illness, but there are different issues for child psychiatry. A source of particular risk in child psychiatry is the number of conflicting obligations. For example, confidentiality $v$. safety or, in 'ethical' terms, autonomy $v$. beneficence; medication - too much or too little; diagnosis entitlement or stigma; the child's interests $v$. the parent's. The context, as usual, is complex, with multi-disciplinary and multi-agency issues. As the assessment and management of risk is a comparatively new paradigm for child psychiatry, there is a lack of published data - a situation that may well change soon. Although laudable efforts are being made to emphasise 'blame-free systems' (Department of Health, 2000) as the safest way forward, political pressures may work in the opposite direction.

\section{Risk: definition and management}

A simple definition of risk is 'the likelihood of something bad happening'. Already, one needs to consider what counts as bad, for whom, in what context and how bad it has to be. Then there is the question of the likelihood. The two together, 'badness' (which may be translatable into cost) and likelihood, will give a guide as to what needs to be prioritised in terms of aiming at risk reduction. An example of this type of thinking is found in the model offered by the Clinical Negligence Scheme for Trusts (http://www.nhsla.com). However, actual preventability and the cost of risk reduction itself should also be considered.

Formal risk management attempts to reduce the risks of adverse events occurring by systematically assessing, reviewing and then seeking ways to prevent their occurrence. The NHS considers clinical risk as one of several risks facing its organisations others include financial, organisational and health and safety. All trusts are required to have risk management strategies in place. Box 1 summarises recommendations for child psychiatrists with regard to the common risks that they face in clinical practice.

\section{What is an error in child psychiatry?}

The terms 'error' or 'mishap' imply both a mistake and consequent harm. However, $\mathrm{p}$-oor practice in, for example, recording or communication does not necessarily lead to adverse outcomes, although it can increase their likelihood. Equally, errors such as the mis-writing of a methylphenidate prescription will be picked up by a vigilant pharmacist, and corrected with only some loss of time and perhaps goodwill. A misdiagnosis - especially not recognising appropriately a serious physical condition suggests not so much that child psychiatrists need to be medical experts, but that they and other staff need to be alert to a variety of possibilities.

Fiona Subotsky is a consultant child and adolescent psychiatrist at the South London and Maudsley NHS Trust, based at the Department of Child and Family Psychiatry (King's College Hospital, Denmark Hill, London SE5 9RS, UK. E-mail: subotsky@clara.co.uk). She has wide managerial experience and has had a particular interest recently in looking at risk issues for child and adolescent mental health services. 


\section{Box 1 Recommendations regarding risks child psychiatry}

- Be aware of standards, guidance and policies at national and local level

- Comply with appraisal/CPD/revalidation requirements, especially ensuring your own appropriate personal development

- Ensure good documentation and communication

- Document a full general assessment, preferably according to a locally agreed template and using a range of informants

- Record risk assessment and management plan - to others, to self and from others

- With high-risk cases especially, ensure that responsibilities of other agencies such as social services or adult mental health services are clarified before closure

- Consider guidance and training on 'appropriate boundaries', to reduce the likelihood of allegations of abuse

- Take care when there is potential conflict between the interests of parents and children and/or when a known pressure group may become involved

- If resource issues such as lack of in-patient facilities are leading to potentially risky management, put them in writing

- Clarification of boundary of professional responsibilities is desirable although difficult

- Confidentiality may be a cause of complaints in both directions (disclosure, non-disclosure): ask the Caldicott Guardian for advice

- Avoid being in a professionally isolated position

- Have your own defence cover, beyond the trust's indemnity

- Membership of the British Medical Association may also be helpful

'Errors' are difficult to evaluate in child mental health as there is considerable variation in practice between and within disciplines and outcomes are usually not immediate, nor necessarily recorded or measured. However, this same situation means that differing views can often be strongly held and sometimes lead to formal complaints by one professional about another.

\section{Types of adverse outcome}

\section{At the individual patient level}

Harm to self

Much of the literature on deliberate self-harm is about overdoses, which are indeed a common presentation to hospital. Other 'suicidal behaviour', such as attempts at hanging, are less common. At the less life-threatening end of the continuum is the phenomenon of self-cutting or burning, and schoolbased staff are likely to know of more than are referred. Teenagers' intent and knowledge of actual lethality may be hard to establish clearly, as may be the degree of formal depression when moodiness and impulsivity seem to predominate. All cases of self-harm need to be taken seriously as there is an increased risk of eventual death by suicide, but the evidence base for effective intervention is sparse (Hawton et al, 2002). Ideally all under- 18 s who have deliberately harmed themselves should be seen and systematically assessed by child mental health professionals and/or trained social workers before discharge, as otherwise follow-up contact is difficult.

There are many instruments for assessing suicide risk in young people, none perfect (Goldston, 2000), but they can be very useful for multi-disciplinary teams in promoting standardised and comprehensive emergency assessments. The major triage decisions are whether the young person can go home safely, whether his or her care needs to be taken over temporarily by social services and whether he or she should be admitted to psychiatric care. Overnight admission is recommended (Royal College of Psychiatrists, 1998), ideally to a paediatric ward or, for 14- to 16-year-olds, to a ward for adolescents, but this is not always possible. For those who can be managed as out-patients, short-term family therapy may reduce suicidal ideation (Byford et al, 1999). Some young people will benefit from treatment of depression, with medication and/or psychotherapy and some, particularly 'repeaters' with unstable emotional disorder, will need to be engaged in longer-term psychotherapy. Misuse of drugs and alcohol merits special inquiry and attention, as does the possibility of abuse.

The majority of young people who harm themselves are girls, but there is a group of highly at-risk young men who may not present to CAMHS because of overdoses and who appear primarily to be delinquent. They have been identified recently through well-publicised deaths at offender institutions and could possibly be helped earlier through the youth offending team system. The suicide rate for 15- to 
19-year-old males, especially by hanging, increased markedly from 1970 to 1998 (McClure, 2001).

Other forms of self-harm include refusal to eat or self-induced vomiting, as with anorexia nervosa and bulimia, reckless behaviour such as may occur with attention-deficit hyperactivity disorder, and use of solvents, drugs and alcohol. Comorbidity is common and underlines the need for full evaluations, both of the child or adolescent and of the family and social circumstances.

\section{Harm to others}

The assessment of risk of harm to others has not previously been clearly part of CAMHS' remit unless in a forensic context. Nevertheless, young people with conduct disorder commonly present not only with a range of antisocial behaviour, but also with physical or verbal aggression in school and/or at home. To inform decision-making, there is again a need to establish what is present within the child, the family and the social circumstances that can be remedied or alleviated. The usefulness of formal risk assessment will depend much on the context, and is helpfully discussed by Sheldrick (1999) and Bailey (2002). Many interventions are best served by close agency collaboration such as through youth offending teams, of which CAMHS may be only a part.

Serious mental illness such as schizophrenia and its attendant risks are of increasing importance as CAMHS takes on responsibility for 16- and 17-yearolds, and admission may be difficult or length of stay short. Early, well-coordinated intervention is essential and is primarily the responsibility of the health service. The Care Programme Approach is an appropriate multi-agency mechanism for this group, and should ease transfer to adult services.

\section{Harm from others}

The risk of harm from others is a familiar concept in CAMHS, and is usually well covered in training on child protection issues. Child and adolescent mental health services can offer help in drawing attention to issues such as parental mental illness, substance misuse and history of violence, as well as being able to provide assessments of parental capacity and attitude, and the emotional and developmental state of the child. Social workers, who have greater expertise and responsibility on this issue, often work within CAMHS teams or are available for advice. Each trust should have its own named child protection doctor and nurse available for consultation. Each case should at least be briefly considered under this heading, even though this may not be the obvious reason for referral. There is a range of sources of possible harm - parents (directly or through failure to protect), other family members, foster carers, other young people or adults, other agency staff (e.g. at school or in a children's home).

The 'child protection' categories are physical abuse, sexual abuse, emotional abuse and neglect. However, presentations are often not as clear-cut as this, and anxieties particularly arise because of potential confidentiality issues, the possible disruption of therapeutic intervention if the situation is formally reported, and because of differences of interest between the child and the adults. Advice should be readily sought and an effective multiagency approach is vital in abuse cases. The CAMHS professionals should be familiar with, and able to contribute to, the Government's child assessment framework (Department of Health et al, 2000), although it is Social Services' responsibility to coordinate the process.

\section{Harm from the health care system and it staff}

Harm to patients from the health care system itself does not usually form an overt part of the individual risk assessment, but is evident through complaints and incident reporting mechanisms and should be considered. Negative effects of treatment would come under this heading: for example, side-effects of medication or adverse effects of in-patient admission such as loss of educational continuity and exposure to further risk. Failure to treat or care effectively is also a risk, sometimes due to lack of resources but also to failure of communication, poor management or incorrect diagnosis. Poor practice with restraint and seclusion procedures has been highlighted, as there have been deaths reported. Some of these have been the result of misguided attempts at 'therapeutic holding' and clear guidelines should be developed by in-patient units similar to those advised by the American Academy of Child and Adolescent Psychiatry (Masters et al, 2002).

Psychotropic medication is used for a variety of indications with children, sometimes 'off licence' owing to lack of evidence regarding safety in paediatric populations. Clinicians need to be aware of prescribing guidelines such as those on stimulants from the National Institute for Clinical Excellence (2000) and the American Academy of Child and Adolescent Psychiatry (Greenhill et al, 2002), and should help to develop local good practice protocols for physical examination, review, information provision and consent.

Abuse by staff is also a possibility and preventive measures are discussed in a report of the Carlile Review (National Association for Wales, 2002), including Criminal Records checking for all staff involved with children, even temporary agency staff. It also makes recommendations regarding the prevention of isolation of staff, including consultants, in in-patient units. Allegations against CAMHS staff 
for transgressing sexual boundaries are not uncommon and are very serious. The Royal College of Psychiatrists' Council Report Vulnerable Patients, Vulnerable Doctors (2002), one of a series on good psychiatric practice, is important training material.

\section{At an organisation and staff level}

Harm to staff can arise directly from a patient or the family and it includes actual physical assault and threatening behaviour. In-patient assaults on staff, even if only minor, are common and need to be prepared for with clear policies, appropriate staffing levels and experience for supervision, early deescalation and access to back up. Threats from parents most commonly occur when there is existing family conflict and possible child abuse. It is now recognised that professional avoidance or appeasement of threatening adults has contributed to serious failures of child protection, so such incidents should be recorded and reported and, where appropriate, shared with other agencies. Community CAMHS should be aware of the need for elementary personal safety precautions, such as not being alone in the evening with clients, asking other team members to be alert when problematic interactions are likely and knowing how to get security support. Possible risks of home and community visiting must also be considered and appropriate policies adopted; these include informing the base where you are going, carrying a mobile telephone and going in pairs. 'Dealing with violence' training needs to be developed specifically for CAMHS.

\section{Risk management and standards}

In theory, risk can be reduced by the setting and monitoring of standards, which if done well should at least help to reduce variability and identify areas for improvement.

General service standards and recommendations relevant to CAMHS are included in the following publications or websites:

- Clinical Negligence Scheme for Trusts (http:// www.nhsla.com)

- Safety First (Department of Health, 2001a)

- Children in Mind: Child and Adolescent Mental Health Services (Audit Commission, 1999)

- Standards for Child and Adolescent Mental Health Services (Finch et al, 2000)

- Managing Deliberate Self-Harm in Young People (Royal College of Psychiatrists, 1998).

- Framework for the Assessment of Children in Need and their Families (Department of Health, 2000)

- Working Together to Safeguard Children (Department of Health et al, 1999).

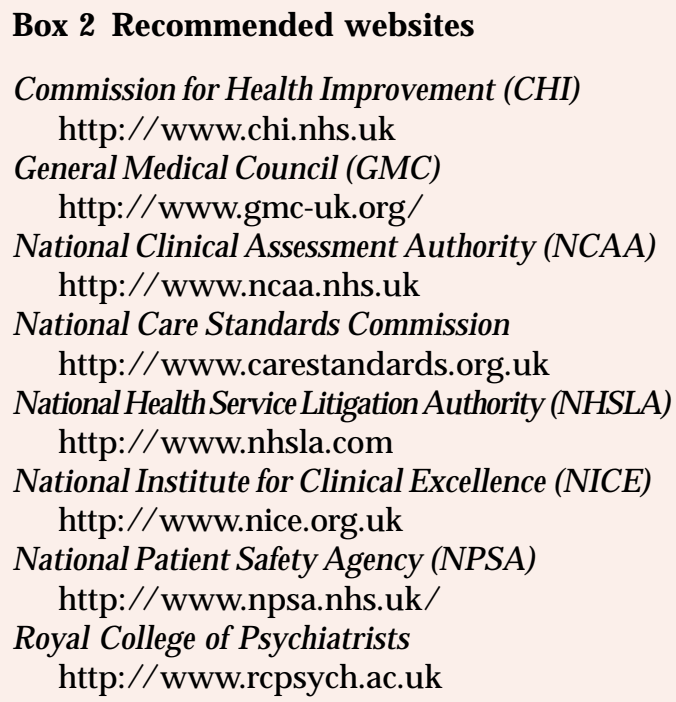

There are also profession-specific standards for child psychiatrists set out in Duties of a Doctor (General Medical Council, 1995) and in Good Psychiatric Practice (Royal College of Psychiatrists, 2000). Apart from their inherent professional value, these are vital documents to be aware of, as others judge us against their contents. Other CAMHS professionals have similar documents and standards that are worth sharing. Box 2 shows the website addresses of agencies that give guidelines or advice relating to clinical risk management.

\section{Trust-level policies}

Child mental health professionals should contribute to local trust policies to ensure their relevance and feasibility for child and adolescent services. In addition they can give important advice on some 'adult' policies, especially in relation to parents with mental illnesses. Important policies include:

- risk management

- child protection

- children visiting

- clinical risk assessment and management of harm

- sexual assault

- management of violence

- restriction

- observation

- 'failure to attend'.

\section{Reporting and monitoring mechanisms}

A panoply of regulatory mechanisms are coming on stream in the UK (Walshe, 2002), some of which are 
mentioned below and in Table 1. Clinical directors in particular need to be aware of their standards, as trusts are subject to considerable pressure for compliance, yet may not be communicating this clearly to potential 'outliers' such as CAMHS.

\section{External inspections}

The Commission for Health Improvement will be progressing shortly to inspections of the clinical governance arrangements of child as well as adult mental health services. The seven areas they look for evidence on are: risk management, clinical audit, staffing and staff management, education, training and lifelong learning, research and effectiveness, clinical information and service user experience. It is likely that most trusts will have committee structures matching these issues. CAMHS need to provide representation and paths of communication, and they will almost certainly need their own systems to consider child mental health issues separately.

The External Clinical Advisory Service of the Royal College of Psychiatrists provides a small multi-disciplinary panel on request of a chief executive or medical director to look at problematic situations and advise. Although the performance of a particular doctor might be at issue, the team looks at the system as a whole, and its remit is different from that of other groups.
General Medical Council (GMC) performance assessment panels visit places of work to investigate the performance of particular doctors, usually as a result of complaints by trusts. The issue must be serious enough to question a doctor's registration.

The National Clinical Assessment Authority (NCAA) was established in April 2001, with the aim of investigating and helping doctors with performance problems at an earlier stage than GMC intervention. Referral will usually be from the trust's medical director. It is hoped that this will reduce the number of suspensions.

\section{External inquiry systems}

Formal external homicide inquiries, although currently under review, still continue, and can include cases in which the perpetrator is a minor. Homicide inquiries have been criticised for their inconsistency of approach, and for their tendency to blame and scapegoat. In addition, lessons are not easily learned from the reports, which are not assembled systematically. An overall analysis from the Zito Trust (Sheppard, 1996) compiled the recommendations then available, but does not report any specifically related to children and adolescents. The National Confidential Inquiry is a different system to which all homicide convictions are reported by jurisdiction of England and Wales,

Table 1 Risks involving children and adolescents, inquiries and risk managment

\begin{tabular}{|c|c|c|}
\hline Fatal adverse events & Inquiry type & Relevant risk management \\
\hline \multicolumn{3}{|l|}{ Harm by others } \\
\hline \multirow[t]{4}{*}{ Homicide/manslaughter } & Internal management inquiry & Child protection policies \\
\hline & Coroner's inquiry & Assessment and recording of risk \\
\hline & $\begin{array}{l}\text { Criminal proceedings against } \\
\text { perpetrator(s) }\end{array}$ & \\
\hline & Possibly Part 8 inquiry & \\
\hline \multicolumn{3}{|l|}{ Self-harm } \\
\hline Suicide/drug overdose & Internal inquiry, sometimes with & Self-harm protocols \\
\hline Self-neglect, e.g. anorexia & independent input & Risk assessment, including the \\
\hline nervosa & Report to confidential inquiry & social context \\
\hline \multirow[t]{3}{*}{ Fatal risk-taking } & Report to NPSA & Comprehensive treatment \\
\hline & & $\begin{array}{l}\text { Specialist treatment (e.g. for anorexia } \\
\text { nervosa) }\end{array}$ \\
\hline & & Access to social intervention \\
\hline \multicolumn{3}{|l|}{ Harm to others } \\
\hline \multirow[t]{4}{*}{ Homicide } & Internal inquiry & Risk assessment \\
\hline & Part 8 or homicide inquiry & Access to specialist forensic opinion \\
\hline & possible & Early intervention for psychosis \\
\hline & & Access to social intervention \\
\hline \multicolumn{3}{|l|}{ Harm from health care system } \\
\hline \multirow{5}{*}{$\begin{array}{l}\text { Treatment death, e.g. from } \\
\text { medication or restraint }\end{array}$} & Part 8 multi-agency review & Evidence-based medication protocols \\
\hline & Internal inquiry with independent & Good recording \\
\hline & input likely & Restraint protocols \\
\hline & Possibly disciplinary proceedings & Training \\
\hline & Coroner's inquiry & \\
\hline
\end{tabular}


Scotland and Northern Ireland (Appleby, 2000) and are followed up to see if there has been mental health service involvement. If so a detailed questionnaire is sent to the trust/consultant concerned. Six per cent of these cases (in England and Wales) were the responsibility of child and adolescent services. Seventeen per cent of cases were thought by the respondents possibly to have been preventable, with compliance and drug and alcohol misuse being major issues. Generally, however, at last contact risk of violence was rarely rated high. These issues will be of increasing relevance to CAMHS as responsibility is taken for older adolescents.

Information on people who die by suicide or who receive an open verdict at a coroner's inquest is obtained from the Office for National Statistics for England and Wales and the General Register Office in Northern Ireland, and suicide information is derived by the National Confidential Inquiry from the General Register Office in Scotland. Again, contact with mental health services is ascertained and questionnaires are sent to the appropriate consultant. Although a substantial proportion of suicides identified were of individuals under 25 years of age, the degree of involvement from CAMHS is not mentioned. The recommendations are so far largely adult-oriented, with special advice on inpatients (such as removal of ligature points), risk training for all staff, careful follow-up and transfer, and appropriate systematic use of the Care Programme Approach. Many of these points are, however, transferable to CAMHS, and progress on them is being externally monitored.

\section{Serious case reviews}

'Serious case reviews', often referred to as 'Part 8 inquiries', are described in the guidance document Working Together to Safeguard Children (Department of Health et al, 1999). When a child dies and abuse or neglect are known or suspected to be a factor, or if there has been extremely serious abuse or harm, agencies should consider whether there are lessons to be learned from an interagency review. The process is led by the Area Child Protection Committee (ACPC) and involves at least the agencies of social services, education, health and the police. Each relevant service undertakes a management review of its involvement with the child and family, and the ACPC produces an overview report and action plan, which is forwarded to the Department of Health (Social Services Inspectorate social care region). Child and adolescent mental health services will be involved directly in only a small proportion of these cases, but should through ACPC membership be actively aware of issues raised and should ensure that the recommendations about good practice are disseminated and acted upon. The recent Department of Health report Learning from Past Experiences - A Review of Serious Case Reviews (Sinclair \& Bullock, 2002) did not identify specific CAMHS involvement in the 40 case reviews sampled. However, issues such as parental mental illness, parental substance misuse and domestic violence were commonly present. Poor assessment, recording and communication were common themes of the reports. Identification of individuals at risk using risk assessment remains uncertain and generally predictability and preventability were thought to be low.

\section{Other external reporting}

As outlined in Building a Safer NHS for Patients (Department of Health, 2001b), the National Patient Safety Agency is now established as a special health authority whose purpose is to coordinate reports of serious adverse incidents and learn from them. 'Mandatory reportables' include: unexpected death while under the direct care of a health professional, unexpected death within 1 month of being seen by a health care professional, suspected homicide by a known mental health service user, suspected suicide (within 1 month of discharge, or following a decision not to admit, or on hospital premises) and alleged rape or sexual assault. Children and young people being seen by CAMHS are at risk of all of these, and the Part 8 system would not pick up all the unexpected deaths as defined in this way.

Serious complaints about psychiatrists as individuals may of course go to the GMC, where if thought by the screeners to be of sufficient importance, they may be further investigated under the headings of Conduct, Health or Performance. Complaints going to the GMC about child psychiatrists and paediatricians especially are sometimes orchestrated by specific campaigns, as indicated in a multi-authored letter to the BMJ (Marcovitch \& COsignatories, 2002). This urges the GMC to be more aware of this type of vexatious complaint, which can lead unwary trusts to suspend as well as to GMC investigations. Riskier areas of child psychiatry in this respect include Munchhausen by proxy syndrome, within-family sexual abuse, custody and access disputes, chronic fatigue, and attention-deficit hyperactivity disorder.

Some complaints may go to the ombudsman if complainants remain dissatisfied with trust procedures. Examples upheld relating to CAMHS are few (D. Hayes, personal communication, 2002), but have included a complaint about a refusal to prescribe a particular medication, a failure to obtain parental consent for home leave and information concerning abuse being circulated to non-clinicians. 
Table 2 Complaints in CAMHS

Type

Access

Boundary issue

Case management

Confidentiality

Medical
Example

Waiting time too long for first appointment

Referral not accepted

Lack of out-of-hours access

Funding delay

Lost referral

Did not receive cancellation letter and attended

Allegation of sexual assault by another patient

Allegation of sexual assault by staff

Inadequate care and management of child following an overdose

Inadequate information given about in-patient

Lack of attention paid to siblings

Too much attention paid to father rather than to patient

Information passed to social services without permission

School given information without permission

Failure to diagnose medical condition
The role of the media should not be forgotten. It is well recognised as a risk by trusts, but less so by individual clinicians. A child psychiatrist's complaint against the BBC was upheld by the Broadcasting Standards Commission, which found that a programme about the treatment of chronic fatigue was unfair and detrimental (Broadcasting Standards Commission, 2001). Litigation against a trust or an individual clinician is also a possibility, and an increasing risk. In the USA the most common malpractice concerns for psychiatrists are the standard of care, record-keeping, confidentiality and responsibility where treatment is shared (Rychik \& Lowenkopf, 2000).

\section{Internal inquiries}

Risk management at an organisational level should include the collation and analysis of incident reports and complaints. These should be examined by the directorate to see what general issues arise beyond the particular case, especially if there are 'clusters'. Local experience suggests that for CAMHS, the most frequently reported incidents are assaults on inpatient wards, and that there is probably underreporting from the community teams. Although complaints are fairly few, they are most common from parents dissatisfied with access to the service or the actual service given, or about confidentiality (Table 2 gives further examples).

Medical disciplinary action by trusts may include suspension, although this is an expensive option. Such information as exists for all specialities suggests that suspension is frequently driven not so much by patient as by professional colleague or managerial complaint, sometimes of a 'face-notfitting' type. Anecdotally, for child psychiatrists this has not infrequently been due to a multi-disciplinary perception of overuse of an authoritarian version of the 'medical model'. Although many cases do not lead to actual dismissal, there is often a prolonged and stressful period of confusion and apparent inaction. It is to be hoped that the NCAA will help prevent or at least resolve more speedily such cases.

\section{Conclusions}

Clinical risk management for child psychiatrists should take place within the clinical governance framework and be developed at CAMHS directorate level, so that it can be implemented by all disciplines and relate to and contribute to adult mental health policies, while having its own specific priorities. Interagency arrangements, especially with social services and youth offending teams, are of great importance as more risk is managed in the community, and these need formal negotiation beyond the individual case, for example for the fulfilment of the Care Programme Approach. Competing interests, such as those between parents and child, lead to difficulties in decision-making and are potential sources of complaint. While trusts should be aware of the variety of risks to their organisation, individual clinicians need also to be aware of the range of personal and professional risks to themselves and take steps to minimise them.

\section{References}

Appleby, L. (2000) Safer services: conclusions from the report of the National Confidential Inquiry. Advances in Psychiatric Treatment, 6, 5-15.

Audit Commission (1999) Children in Mind: Child and Adolescent Mental Health Services. London: Audit Commission Publications. 
Bailey, S. (2002) Violent children: a framework for assessment. Advances in Psychiatric Treatment, 8, 97-106.

Broadcasting Standards Commission (2001) Fairness Adjudication Summaries (Bull. 40): http://www.bsc.org.uk

Byford, S., Harrington, R., Torgerson, D., et al (1999) Costeffectiveness analysis of a home-based social work intervention for children and adolescents who have deliberately poisoned themselves. Results of a randomised controlled trial. British Journal of Psychiatry, 174, 56-62.

Department of Health (2000) An Organisation with a Memory. Report of an expert group on learning from adverse events in the NHS chaired by the Chief Medical Officer. London: Stationery Office.

- (2001a) Safety First: Five-Year Report of the National Confidential Inquiry into Suicide and Homicide by People with Mental Illness. London: Department of Health.

- (2001b) Building a Safer NHS for Patients. http://www. doh.gov.uk/buildsafenhs

- Home Office \& Department for Education and Employment (1999) Working Together to Safeguard Children. London: Stationery Office.

- Department for Education and Employment \& Home Office (2000) Framework for the Assessment of Children in Need and their Families. London: Stationery Office.

Finch, J., Hill, P. \& Clegg, C. (2000) Standards for Child and Adolescent Mental Health Services. London: Health Advisory Service.

General Medical Council (1995) Duties of a Doctor: Guidance from the General Medical Council. London: GMC.

Goldston, D. B. (2000) Assessment of Suicidal Behaviors and Risk Among Children and Adolescents. Bethesda, MD: NIMH.

Greenhill, L. L., Pliszka, S., Dulcan, M. K., et al (2002) Practice parameter for the use of stimulant medications in the treatment of children, adolescents and adults. Journal of the American Academy of Child and Adolescent Psychiatry, 41 (suppl. 2), 26S-49S.

Hawton, K., Townsend, E., Arensman, E., et al (2002) Psychosocial and pharmacological treatments for deliberate self-harm. Cochrane Library, issue 3. Oxford: Update Software.

Marcovitch, H. \& co-signatories (2002) GMC must recognise and deal with vexatious complaints fast (letter). $B M J$ 324, 167-168.

Masters, K. J., Bellonci, C., Bernet, W., et al (2002) Practice parameter for the prevention and management of aggressive behaviour in child and adolescent psychiatric institutions, with special reference to seclusion and restraint. Journal of the American Academy of Child and Adolescent Psychiatry, 41 (suppl. 2), 4S-25S.

McClure, G. M. G. (2001) Suicide in children and adolescents in England and Wales 1970-1998. British Journal of Psychiatry, 178, 469-474.

National Assembly for Wales (2002) 'Too Serious a Thing': The Review of Safeguards for Children and Young People Treated and Cared for by the NHS in Wales. (Carlile Review). Cardiff: National Assembly for Wales.

National Institute for Clinical Excellence (NICE) (2000) Guidance on the Use of Methylphenidate (Ritalin, Equasym) for Attention Deficit/Hyperactivity Disorder (ADHD) in Childhood. London: NICE.

Royal College of Psychiatrists (1998) Managing Deliberate Self-Harm in Young People (Council Report CR64). London: Royal College of Psychiatrists.

- (2000) Good Psychiatric Practice 2000 (Council Report CR83). London: Royal College of Psychiatrists.

- (2002) Vulnerable Patients, Vulnerable Doctors. Good Practice in our Clinical Relationships (Council Report CR101). London: Royal College of Psychiatrists.

Rychik, A. M. \& Lowenkopf, E. L. (2000) Reviewing Medical Malpractice and Risk Management Issues. Psychiatric Times, XVII, Issue 8.

Sheldrick, C. (1999) The assessment and management of risk in adolescents. Journal of Child Psychology and Psychiatry, 40, 507-518.
Sheppard, D. (ed.) (1996) Learning the Lessons (2nd edn). London: The Zito Trust.

Sinclair, R. \& Bullock, R. (2002) Learning from Past Experience - A Review of Serious Case Reviews. London: Department of Health.

Walshe, K. (2002) The rise of regulation in the NHS. BMJ, 324, 967-970.

\section{Multiple choice questions}

\section{A 15-year-old girl presenting to a hospital A\&E after} an overdose:

a should be sent home after triage by A\&E staff

$\mathrm{b}$ is unlikely to be at risk of later suicide

c may benefit from family therapy

$d$ is more likely than not to benefit from antidepressant medication.

2 Introducing clinical risk management to CAMHS:

a has few training implications for the multi-disciplinary team

b implies a radical change from standard child psychiatric practice

c is met by incident reporting systems

$\mathrm{d}$ is likely to require the development of protocols.

3 A 15-year-old in the care of the local authority hangs himself 1 week after discharge from a psychiatric unit. The incident:

a should be the subject of a Section 15 inquiry

b should be reported to the National Patient Safety Agency

c should be reported to the GMC

d will be looked at from the point of view of the 'Safety First' recommendations.

4 Common adverse incidents in CAMHS include:

a homicide

b deliberate self-harm

c assaults on in-patient staff

$\mathrm{d}$ medication errors.

5 Child psychiatrists:

a should always share information freely

b need not be concerned with risks presented to others if there is no formal mental illness

c should discuss confidentiality issues with the Caldicott guardian

$\mathrm{d}$ should be able to defend their case management actions with evidence.

\section{MCQ answers}

$\begin{array}{llllllllll}1 & & 2 & & 3 & & 4 & & 5 & \\ \text { a } & \text { F } & \text { a } & \text { F } & \text { a } & \text { F } & \text { a } & \text { F } & \text { a } & \text { F } \\ \text { b } & \text { F } & \text { b } & \text { F } & \text { b } & \text { T } & \text { b } & \text { T } & \text { b } & \text { F } \\ \text { c } & \text { T } & \text { c } & \text { F } & \text { c } & \text { F } & \text { c } & \text { T } & \text { c } & \text { T } \\ \text { d } & \text { F } & \text { d } & \text { T } & \text { d } & \text { T } & \text { d F } & \text { d T }\end{array}$

